\title{
Abordaje Clínico de un Incisivo Maxilar Permanente con Fractura Corono Radicular Compleja Mediante Reimplante Intencional
}

\author{
Clinical Approach of Permanent Maxillary Incisor with \\ Complicated Crown-root Fracture Through Intentional Replantation
}

Jaime Díaz M."; Viviana Barrera O."; Alejandra Jans M.." \& Carlos Zaror S."

DÍAZ, M. J.; BARRERA, O. V.; JANS, M. A. \& ZAROR, S. C. Abordaje clínico de un incisivo maxilar permanente con fractura corono radicular compleja mediante reimplante intencional. Int. J. Odontostomat., 8(2):299-307, 2014.

RESUMEN: EI manejo de las fracturas corono radiculares es un desafío clínico interdisciplinario, tanto en su atención de urgencia como en la planificación y ejecución del tratamiento definitivo. Se describen tres alternativas principales como terapia: tracción quirúrgica u ortodóncica del remanente corono radicular, gingivectomía y reimplante intencional dependiendo algunos factores como: edad del paciente, condición ligamento periodontal, longitud radicular, extensión del rasgo e fractura, entre otros. Paciente de sexo masculino de 10 años, con trauma dentoalveolar complejo, donde destaca la fractura corono radicular, diente 11, que presenta extensión subgingival mayor a $5 \mathrm{~mm}$. Dada la complejidad de la situación clínica, se optó por el reimplante intencional, para permitir la reposición del fragmento con técnica adhesiva extraoral. Luego de los primeros dieciséis meses de seguimiento se observa una adecuada cicatrización periodontal y un resultado estético aceptable. El reimplante intencional puede ser considerado un adecuado tratamiento en casos de fracturas corono radiculares complejas, cuando el rasgo de fractura se encuentra ubicado en zonas que impiden la utilización de otras técnicas, para su rehabilitación. De igual manera se permite la mantención de la altura y volumen del hueso alveolar de pacientes en crecimiento.

PALABRAS CLAVE: reimplantación de dientes intencional, fractura coronaria en niños, reinserción coronal.

\section{INTRODUCCIÓN}

En los últimos años ha existido un mayor conocimiento e investigación sobre trauma dentario, el cual corresponde aproximadamente al $5 \%$ de todas las patologías que solicitan atención de urgencia. En la actualidad, y de acuerdo a varios estudios epidemiológicos, se sabe que el trauma dentoalveolar es una patología muy frecuente en niños y adolescentes, especialmente entre 7 y 14 años (Marcenes et al., 2000; Glendor, 2008).

Una fractura corono-radicular (FCR) se define como "la lesión traumática de los tejidos dentarios duros que involucra de manera simultánea la porción coronaría y radicular, comprometiendo el esmalte, dentina, y cemento, las que pueden ser agrupadas de acuerdo al compromiso y exposición pulpar en complicadas y no complicadas" (Andreasen et al., 2010). La causa más común es un trauma directo, en el que la dirección de la fuerza impactante determina la orientación en bizel del rasgo de fractura, normalmente desde vestibular hacia palatino con extensión subgingival e infraósea.

Dentro de las alternativas de tratamiento para FCR descritas en la literatura se encuentran: (1) eliminación del fragmento fracturado en caso de fracturas superficiales, (2) extrusión ortodóncica del fragmento apical (3) extrusión quirúrgica del fragmento apical, y

\footnotetext{
Profesor Asociado, Director Especialidad de Odontopediatría, Facultad de Odontología, Universidad de la Frontera, Temuco, Chile * Alumna especialidad Odontología Pediátrica, Universidad de la Frontera, Temuco, Chile.

*** Profesor Asistente, Magister en Epidemiología Clínica, Académico Especialidad de Odontopediatría, Facultad de Odontología, Universidad de la Frontera, Temuco, Chile.
} 
(4) gingivectomía. La elección de la mejor alternativa va a depender de algunos factores como, longitud y estado de desarrollo radicular, compromiso estético, edad del paciente, profundidad y ubicación del rasgo de fractura, costo, habilidad y experiencia de quien realizará la intervención, entre otros. En los últimos años se ha considerado otra alternativa de tratamiento: el reimplante intencional (RI), el cual ha sido considerado como confiable debido a la alta capacidad de respuesta regenerativa del ligamento periodontal (LPD). El objetivo del RI es reimplantar el diente dentro de su alvéolo en su posición original, luego de ser intervenido extraoralmente (Tsukiboshi, 2002; Kim et al., 2013).

El objetivo de este reporte es mostrar el tratamiento y la evolución de un paciente en edad escolar con FCR compleja en incisivo central maxilar, tratado con reimplante intencional, y discutir los fundamentos de esta terapia.

\section{REPORTE DE CASO}

Paciente de 10 años 8 meses de edad, sexo masculino, sin antecedentes médicos relevantes, que solicita atención de urgencia por traumatismo dentoalveolar (TDA), producto de un accidente de tránsito, en Septiembre del 2011. Fue estabilizado en el Servicio de Urgencia Infantil del Hospital Hernán Henríquez Aravena de Temuco (HHHA), e intervenido en pabellón 24 horas después del accidente.

Cuarenta y ocho horas después, fue derivado al Servicio de Odontopediatría con el siguiente diagnóstico inicial: traumatismo dentoalveolar complejo que consistió en Intrusión con gran desplazamiento hasta bajo la espina nasal anterior de ambos incisivos centrales maxilares con fractura no complicada de la corona, fractura de tabla ósea bucal del proceso alveolar entre ambos incisivos centrales maxilares; múltiples heridas cortantes, erosiones en labio inferior y región facial (Fig. 1). El procedimiento de urgencia para su estabilización consistió en: reposición quirúrgica con fórceps de dientes 11 y 21 ; sutura de tejidos blandos y estabilización dentaria con un férula flexible de alambre trenzado y composite desde diente 54 a diente 64 , hasta decidir por un tratamiento definitivo (Fig. 2). Luego de su estabilización y posterior derivación, al examen clínico se observó muy mala higiene, edema gingival y halitosis; se detectó además rasgo de fractura vertical con extensión subgingival mayor a $5 \mathrm{~mm}$ en cara palatina de diente 11, compatible con FCR compleja; fragmento palatino móvil y desplazado.

Debido a la severidad, naturaleza y características de la lesión traumática, y presencia de un desarrollo radicular prácticamente completo en el diente comprometido que implica la interrupción del aporte vascular pulpar, imposibilitando una real futura revascularización, se indica terapia endodóntica con hidróxido de Calcio en diente 11 y 21 . Se retira el fragmento palatino del diente 11 , y se mantiene almacenado en suero fisiológico. Al retirar el fragmento coronal de la FCR, se logra apreciar la real profundidad de la extensión subgingival del rasgo de fractura (Fig. 3).

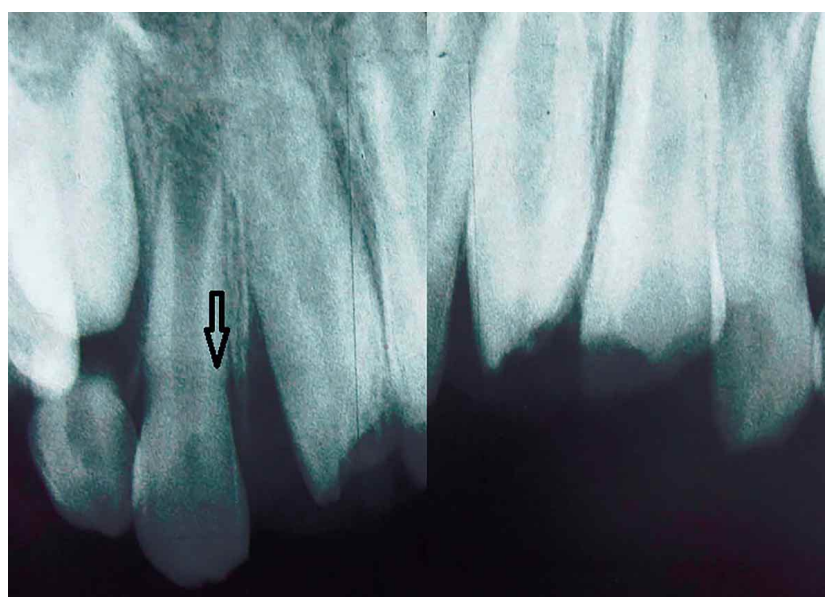

Fig. 1. Visión radiográfica al momento de la atención de urgencia. Se observa el desplazamiento axial severo de ambos incisivos centrales 11 y 21 . Nótese además que en diente 12 la unión esmalte-cemento está hacia apical en relación a límite de cresta ósea marginal, lo cual es indicativo de luxación intrusiva.

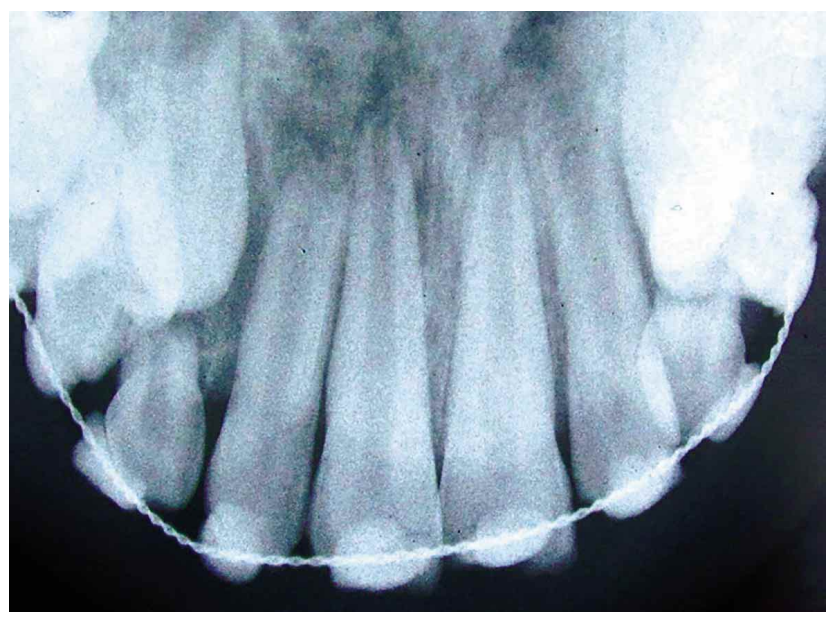

Fig. 2. Control radiográfico inmediato; se observan dientes 11 y 21 reposicionados y estabilizados con férula de alambre y composite. 


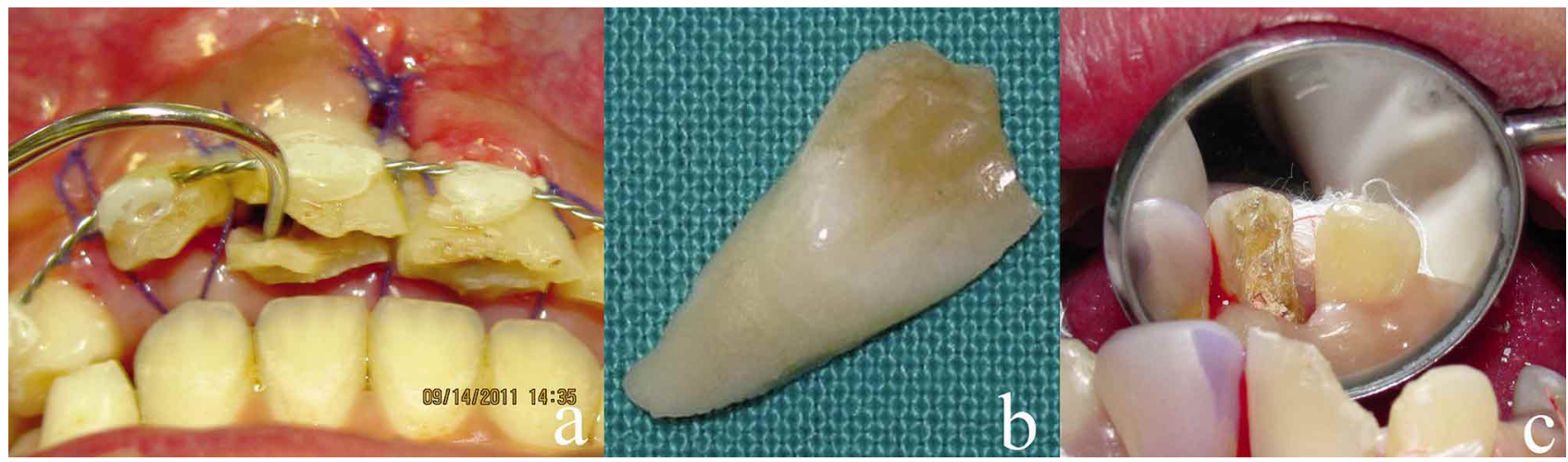

Fig. 3. a) Vista clínica frontal; destaca rasgo de fractura coronaria en sentido mesio-distal con dirección axial y fragmento corono radicular con movilidad extrema. b) Fragmento palatino; se puede apreciar la gran extensión radicular de la cara palatina de la corona del diente 11. c) Vista palatina de diente 11; destaca la gran extensión subgingival de la fractura coronoradicular. Debido a la dificultad en su rehabilitación, se decide por reimplante intencional.

Posterior a tratamiento de endodoncia, y considerando la gran extensión subgingival de la fractura, se planifica el RI del diente 11, bajo los siguientes fundamentos: (i) observamos presencia de hiperplasia gingival que favorece mayor acúmulo de biofilm y abundante hemorragia, (ii) longitud radicular adecuada (iii) hay un rasgo de fractura localizado a más de $5 \mathrm{~mm}$ de profundidad. Sin embargo, a pesar de la longitud radicular favorable, la tracción quirúrgica u ortodóncica necesariamente expondrá una amplia extensión de superficie radicular, lo que va en desmedro de un resultado funcional y estético adecuado.

En este contexto, considerando la alta capacidad de reparación del LPD, se decide por el RI del diente 11, para permitir la ejecución de la técnica de reposición y adhesión de los fragmentos de manera extra alveolar.

Procedimiento clínico: reimplante intencional. Bajo anestesia local (Alphacaine $100 \circledR D F L$, Brasil), se realiza la luxación y extracción del fragmento intraalveolar del diente 11, con movimientos suaves, atraumáticos y rotatorios, para lograr mantener la máxima integridad del LPD adherido a la superficie del cemento radicular. En forma paralela un segundo operador, prepara el fragmento corono radicular almacenado previamente en suero fisiológico, para ser reposicionado usando técnica adhesiva. Se aplica ácido Ortofosfórico 35\% (UItra-Etch ${ }^{\circledR}$ Etchant, Ultradent, Utah, USA) durante 15-30 segundos en ambos fragmentos, el segundo paso consiste en la aplicación de adhesivo para resina (AdperTM single Bond 2, 3M ESPE ${ }^{\circledR}$, MN, USA), y composite restaurador universal (Filtek TM Z - 250, 3M ESPE) en el diente extraído, para lograr la unión y asentamiento de ambos fragmentos. Se elimina el exceso de material y se polimeriza por $40-60$ segundos desde la superficie bucal y palatina. Durante el procedimiento de adhesión, se debe ser muy riguroso y cuidadoso para evitar la manipulación de la superficie radicular, y por lo tanto para evitar producir mayor daño a las células del LPD; se opta por mantener el diente sostenido a nivel cervical con el fórceps (Fig. 4a).

El tiempo extraoral del diente 11 correspondió a 9-10 minutos, lo cual es considerado apropiado. Posteriormente se reimplanta en su alvéolo con suave y cuidadosa presión digital. Se procede a suturar papilas gingivales (Seda Quirúrgica trenzada no absorbible $5 / 0$, NC Braun ${ }^{\circ}$ Aesculap) e inmediata reconstrucción del ángulo distal con corona preformada de celuloide (TDV Dental Ltda., Brasil) y composite (Fig.4b).

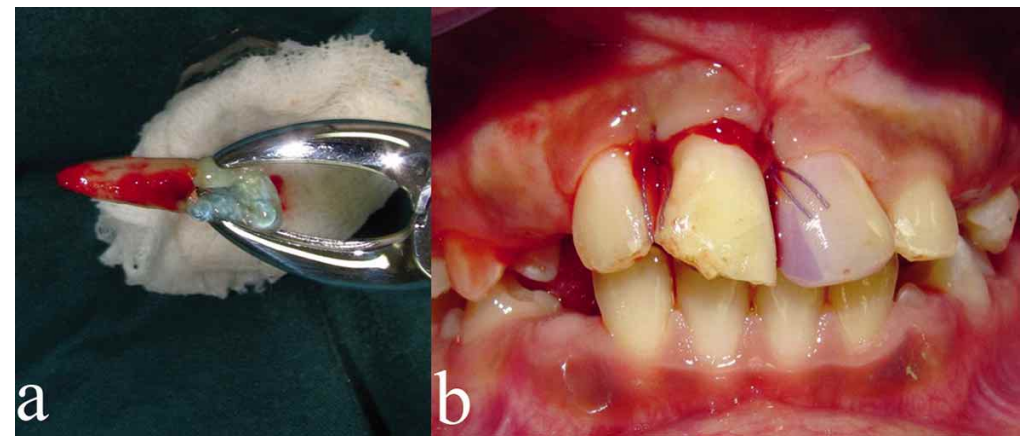

Fig. 4. a) Exodoncia diente 11 y grabado ácido de la porción coronaria fracturada, se observa superficie radicular hemorrágica lo cual corrobora la presencia de LPD vital. b) Visión clínica inmediata post reimplante intencional de diente 11 con fragmento reposicionado. 
Se estabilizó el diente autotransplantado con férula fisiológica de alambre de ligadura trenzada (Stain Less Steel T-304 L Ligature Wire 0,10") más composite, y se realizó aseo quirúrgico con solución de clorhexidina al 0,12\% (Oralgene ${ }^{\circledR}$ Lab. Maver Chile). Se indicaron los cuidados de rigor: dieta blanda por 2 semanas, aseo bucal con cepillo suave 3-4/día con la ayuda de clorhexidina. No fue necesaria la indicación de antibióticos.

Controles clínicos y radiográficos. Al control de los siete días se observó una evolución satisfactoria, ausencia de dolor y prueba de percusión normal. A los 14 días se retiró la férula, radiográficamente no se evidenciaron signos ni síntomas clínicos de complicaciones en la cicatrización periodontal y ósea (Fig. 5a). El paciente se mantuvo en buenas condiciones generales, a pesar del inadecuado control del biofilm. Sin embargo tres meses después, se evidenció clínicamente signos de infraoclusión del diente 1.2 y ruido metálico a la prueba de percusión compatible con reabsorción por reemplazo (Fig. 5b).

Cinco meses luego del reimplante, se apreció clínicamente mayor infraoclusión del diente 12, lo cual afectó claramente la función y aspecto estético del diente 11 reimplantado (Fig. 5c). Se realizó control con tomografía computarizada Cone - Beam (PaxZenith 3D, Vatech Co. Ltd., Gyeonggi-Do, Korea; 2010), el cual mostró con mayor detalle imagen tridimensional y diferentes aspectos sobre cambios estructurales y el proceso de cicatrización en diente 11. Se logró apreciar además un adecuado asentamiento del fragmento adherido, y el defecto óseo producido por la reabsorción por reemplazo del diente 12 (Fig. 6). El paciente se fue mantenido en controles clí-

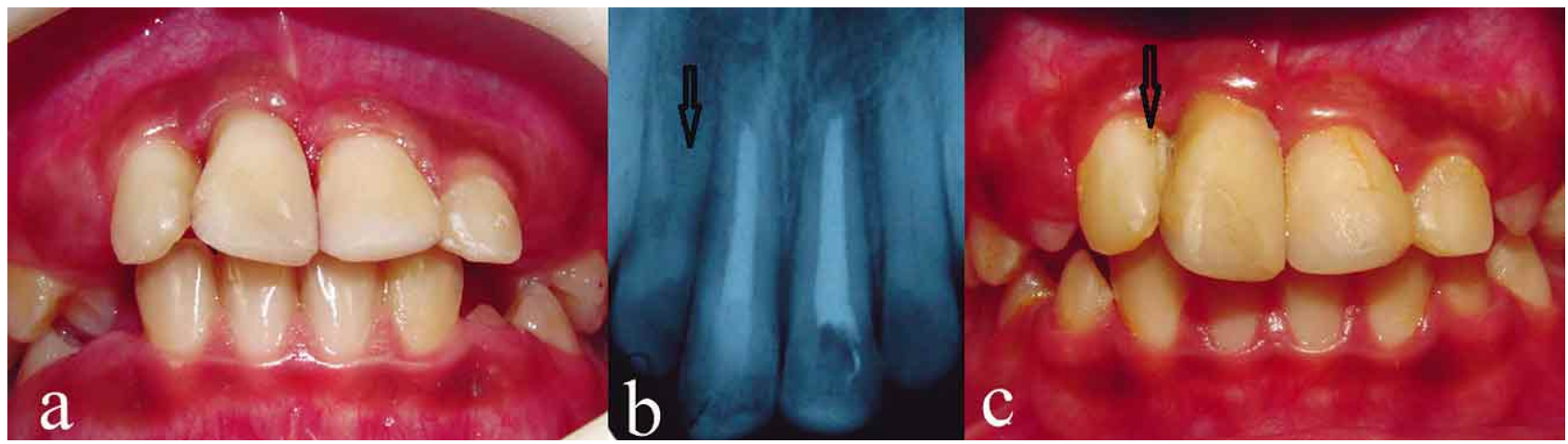

Fig. 5. a) Aspecto clínico después de 2 semanas; la férula es retirada. Diente 11 asintomático al test de percusión y palpación del vestíbulo. b) Control radiográfico posterior a 3 meses. Diente 11 presenta condiciones oseo periodontales adecuadas, en cambio diente 12 con claros signos radiográficos de reabsorción por reemplazo, con ausencia de lámina dura y de espacio periodontal. Notese diente 22 con obliteración casi completa del conducto radicular, asociado a una óptima cicatrización pulpar. c) Cinco meses de evolución, diente 11 con RI mantiene las condiciones favorables en cuanto a cicatrización periodontal; sin embargo se aprecia mayor grado de infraoclusión del diente 12 debido al desarrollo de reabsorción por reemplazo (anquilosis).

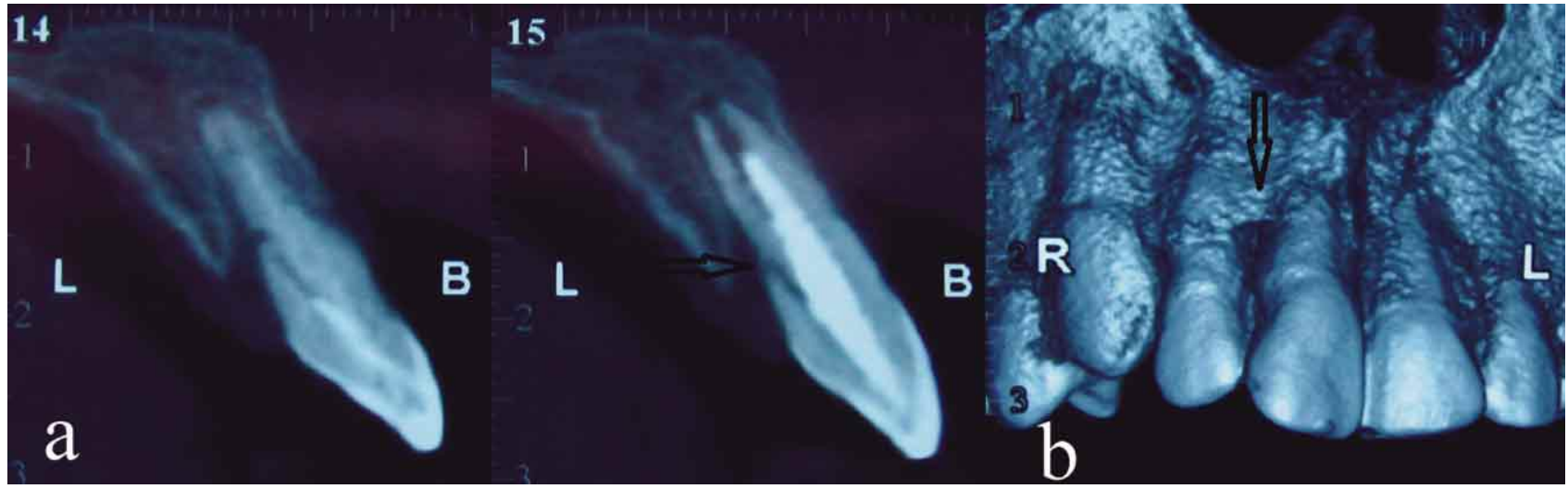

Fig. 6. a) Estudio con cortes de 0,5 mm que muestra la extensión, dirección y ubicación del rasgo de fractura en diente 11. Se comprueba en la dirección oblicua del rasgo de fractura hacia la cara palatina de la superficie radicular. b) Tomografia computarizada reconstrucción 3D; se aprecia defecto óseo entre dientes 12 y 11 asociado a reabsorción por reemplazo de diente 12. 
nicos regulares; 16 meses posteriores al reimplante intencional del diente 11, éste no presentó signos clínicos ni radiográficos de reabsorción radicular externa, ha mantenido condiciones funcionales óptimas y una adecuada salud gingival.

\section{DISCUSIÓN}

Las lesiones dentarias traumáticas en niños y adolescentes con dentición permanente joven necesariamente comprometen 3 aspectos básicos como la función, estética y autoestima, lo cual afecta la calidad de vida (Cortes et al., 2002; Ramos-Jorge et al., 2007). Otros estudios confirman la importancia de realizar un tratamiento adecuado a niños y escolares con traumatismos dentarios, ya que estos se ven afectados notoriamente en sus funciones orales, el bienestar emocional y social (Traebert et al., 2012).

Actualmente, estudios epidemiológicos sobre traumatismos dentarios los consideran una de las patologías más frecuentes en niños y adolescentes, especialmente en edad escolar entre 7 y 14 años, lo cual aumenta las probabilidades de enfrentarse a pacientes adolescentes con injurias dentarias, asociadas a actividades deportivas, caídas y accidentes en bicicleta (Marcenes et al.; Glendor, 2008). Las fracturas que comprometen de manera simultánea la porción coronaria y radicular está muchas veces asociada a estas causas, y se considera por tanto un importante factor que complica el escenario clínico.

Una de las lesiones traumáticas dentoalveolares que implica un mayor desafío en su planificación y tratamiento son las FCR debido a que: (1) existe poca experiencia en el manejo de FCR, (2) su prevalencia es baja, la que según estudios epidemiológicos corresponden aproximadamente a menos del $2 \%$ de todas las lesiones traumáticas de los tejidos dentarios duros, y (3) requiere necesariamente la participación de un equipo multidisciplinario (Cortes et al.; De Castro et al., 2010).

Según su severidad, las FCR comprometen diversas estructuras del diente como son esmalte, dentina, cemento y pulpa; sin embargo el tratamiento y pronóstico está influenciado también por la dirección del rasgo de fractura la cual según la fuerza del impacto y su vector, se obtendrá una fractura corono radicular vertical, oblicua o múltiples fracturas que involucran corona y raíz (Glendor, 2009).
Estudios que miden el conocimiento de especialistas en Rehabilitación oral sobre el adecuado manejo de fracturas coronarias y FCR, muestran que elaborar un plan de tratamiento en el caso de FCR complicadas y no complicadas es usualmente desafiante, especialmente cuando el rasgo de fractura se extiende bajo el nivel gingival e incluso más allá del nivel óseo. El tratamiento propiamente tal y la recuperación de la función y estética constituye lo más complejo, y requiere de un equipo multidisciplinario preparado y actualizado para proveer la mejor alternativa terapéutica (De Castro et al.; Sockalingam \& Mahyuddin, 2009). Por lo tanto, dado la complejidad de la lesión es necesario la participación de especialistas de diferentes áreas; Odontopediatría, Ortodoncia, Cirugía, Endodoncia, Periodoncia y Rehabilitación.

La primera aproximación clínica es la evaluación de urgencia, la cual corresponde a la estabilización temporal del fragmento corono radicular a los dientes vecinos, junto a la evaluación radiográfica, para permitir la adecuada planificación posterior del tratamiento y favorecer el bienestar del paciente (Diangelis et al., 2012). De esta manera fue enfocado el tratamiento de urgencia en este reporte de caso, en que el paciente fue estabilizado, ferulizado y posteriormente enviado a la Especialidad de Odontopediatría para su evaluación, control y determinar la planificación del tratamiento posterior.

Para la rehabilitación adecuada de un diente permanente con FCR la literatura especializada establece algunas alternativas de tratamiento como: (1) Remoción del fragmento coronario y restauración del diente (con el mismo fragmento fracturado, con composite o ambas), si la línea de fractura no involucra el espacio biológico, lo que corresponde de manera muy general, a la unión dentogingiva, (2) Remoción del fragmento coronario, complementado con gingivectomía u osteotomía para exponer el rasgo de fractura previo a la restauración. (3) Remoción del fragmento coronario, tratamiento endodóntico y restauración del diente con prótesis fija singular (PFS). (4) Remoción del fragmento coronario, tratamiento endodóntico y luego tracción ortodóncica o quirúrgica del fragmento apical, (5) Reimplante intencional, (6) Exodoncia y reemplazo protésico. (7) Exodoncia y cierre ortodóncico (Diangelis et al.; Sockalingam \& Mahyuddin).

La rehabilitación estética y funcional es el principal objetivo en el tratamiento de FCR. Para esto, se debe considerar que la reposición del fragmento del diente fracturado con técnicas adhesivas, es una bue- 


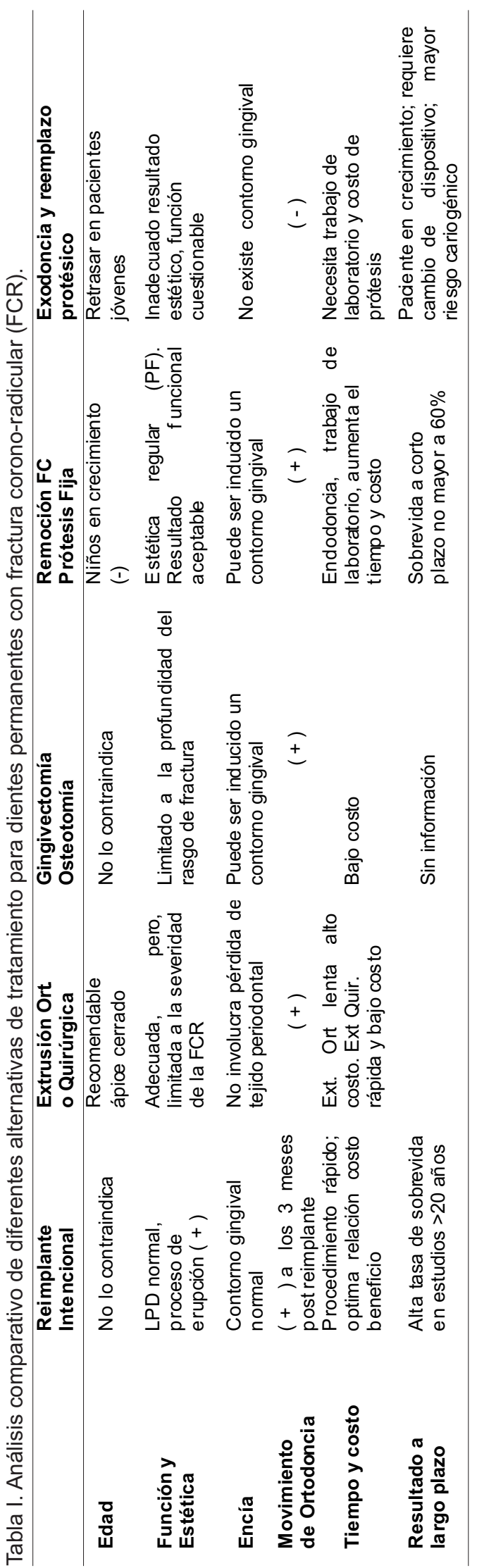

na alternativa y la más conservadora. La literatura describe múltiples ventajas de la reposición del fragmento coronario: mejor resultado estético estéticos en cuanto a color y morfología que no se puede lograr con ningún material de restauración, mantiene la función incisal en la estructura dental, adecuada resistencia al desgaste, desgaste incisal coincide con el desgaste del diente adyacente, buena aceptación por el paciente, menor número de sesiones de tratamiento y menor costo comparado con otras alternativas terapéuticas (Demarco et al., 2004; Rappelli et al., 2002). Considerando lo anterior, el reimplante intencional, definido como el procedimiento quirúrgico en el cual el diente comprometido es extraído, intervenido fuera de la cavidad oral para realizar la reposición y adhesión del fragmento coronario y luego reimplantado en su alvéolo (Peer 2004; Tsukiboshi), es considerado una alternativa viable en el manejo de FCR cuando la línea de fractura se extiende bajo el margen gingival u óseo, y en la cual la cirugía periodontal no es recomendada por razones estéticas (Dogan et al., 2013), situación considerada y analizada de acuerdo a las características del paciente reportado en este caso.

Otros autores mencionan el reimplante intencional con la variante de rotación en $180^{\circ}$, y señalan que es el tratamiento que "lidera" en cuanto al resultado clínico, ya que un rasgo de fractura subgingival en palatino se convierte en una línea supragingival en vestibular, permitiendo mejores resultados estéticos al tener un adecuado acceso al rasgo de fractura (Kim et al.; Fariniuk et al., 2003).

Algunas de las ventajas del RI al compararlo con otras alternativas son las siguientes: (1) Ocupa menos tiempo clínico que otras técnicas (2) Logra mayor precisión en la identificación de la línea de fractura después de la extracción (3) Puede mantener las propiedades estéticas del diente fracturado y del diente adyacente (Kim et al.).

Diversos factores influyen en la elección de la mejor alternativa de tratamiento en casos de FCR como: (1) Edad del paciente, (2) Nivel de función y estética, (3) Condiciones periodontales, (4) Diagnóstico de dientes vecinos, (5) Tiempo transcurrido desde el accidente, (6) Costos del tratamiento y (7) Resultados a largo plazo. Las publicaciones que describen el reimplante intencional como alternativa en el tratamiento de FCR, mencionan que no existe límite de edad para realizar el procedimiento, permite mantener LPD normal, es posible continuar con el proceso de erupción, mantener el volumen de reborde alveolar, mantener un buen contorno gingival, permite movimientos de ortodoncia posteriores, es un procedimiento rápido y de bajo costo. Actualmente existen múltiples estudios con seguimiento a largo plazo mayor a 30 años en casos de transplantes dentarios, en los cuales los fundamentos y bases biológicas de cicatrización periodontal son los mismos que para los casos de RI que se consideraron exitosos, al no presentar - presentar una muy baja frecuencia de complicaciones postoperatorias como reabsorción radicular, ya sea por reemplazo 
o inflamatoria (Park et al., 2011; Zachrisson et al., 2004; Czochrowska et al., 2002). En la Tabla I, se muestra de manera resumida los criterios a considerar para la elección de diferentes alternativas de tratamiento para dientes permanentes con FCR.

Aunque el RI ha descrito un número importante de ventajas comparado a otras técnicas, su mayor desventaja es el potencial desarrollo de reabsorción radicular en el post-operatorio, que incluye la reabsorción por reemplazo, la reabsorción radicular inflamatoria, y en una muy menor proporción la reabsorción cervical invasiva. Durante el procedimiento del RI se produce una lesión mecánica sobre el LPD y superficie de cemento radicular, resultando en una respuesta inflamatoria localizada. Si no existen nuevos estímulos inflamatorios, ni mayor estimulación de la actividad mediada por osteoclastos, la cicatrización del LPD ocurre dentro de un período no mayor a 2-3 semanas (Andreasen \& Hjorting-Hansen, 1966).

El desarrollo de reabsorción radicular inflamatoria, ocurre en respuesta a la infección bacteriana intracanal radicular, lo cual produce una lesión combinada del tejido pulpar, periodontal y ósea, es decir, se produce un proceso inflamatorio masivo que amplifica la reabsorción radicular. Para minimizar o evitar esta complicación, en el caso informado, se realizó previamente terapia de endodoncia, la cual estaba además indicada dado las características de la lesión traumática inicial y a que el diente comprometido presentaba ya formación radicular casi completa. En el peor de los escenarios, si el RI fracasa, se presenta de todas formas una situación o escenario favorable debido a la presencia de un área receptora adecuada en cuanto a condiciones y características del volumen y altura del reborde alveolar para una futura rehabilitación con implantes, lo cual fue considerado en la planificación del tratamiento de este caso reporte.

EI RI debe ser planificado y ejecutado de manera acuciosa para evitar perdida de células del LPD. El procedimiento de la extracción, reimplante, forma de manipulación y preservación del diente es de crucial importancia para mantener la viabilidad de las células del LPD, y evitar un daño severo sobre la superficie radicular. Con esas consideraciones, en el caso informado, el tiempo extraoral del diente reimplantado no fue mayor a $9 \mathrm{mi}-$ nutos, no se manipuló la superficie radicular, ya que el diente estuvo siempre sostenido con fórceps a nivel del cuello anatómico y al mismo tiempo suavemente apoyado sobre una gasa estéril embebida de solución fisiológica para evitar la deshidratación celular.
El alargamiento coronario está indicado para el restablecimiento del ancho biológico en casos de FCR, en la cual el margen de la fractura está cercano al nivel óseo; sin embargo es compleja su aplicación en la zona anterior por motivos que se ve afectada la estética (Baratieri et al., 1993). La extrusión quirúrgica u ortodóncica logra mantener una función y estética adecuada. Sin embargo, esta técnica debe ser sometida a un profundo análisis crítico cuando es el tratamiento de elección en conjunto con una reposición de fragmento, ya que se altera significativamente la línea oclusal e incisal, dando como resultado un pobre aspecto estético. Es decir, esta técnica se encuentra limitada a la localización, extensión y dirección del rasgo de fractura, ya que si se encuentra más allá del $1 / 3$ medio radicular, al realizar la extrusión el diente quedará con una inadecuada relación corona/raíz perjudicando su estabilidad, además de ser una alternativa antiestética (Sockalingam \& Mahyuddin; Saito et al., 2009; Oesterle \& Wood, 1991). Al analizar la alternativa protésica en un paciente joven o adolescente, ya sea carillas o prótesis fija singular en incisivos permanentes maxilares, la evidencia señala que es por lo menos cuestionable porque: (1) Existe sacrificio de estructura sana del diente (2) Gran tamaño del tejido pulpar (3) Existe una erupción progresiva (4) Inestabilidad del margen gingival ,(5) Alto costo, y (6) tazas de sobrevida bajas a corto y mediano plazo (Czochrowska et al.).

Según lo expuesto, al comparar con otras posibles técnicas, facilitar la adhesión del fragmento al diente por medio del reimplante intencional, fue considerado una alternativa práctica, estética y de bajo costo para el paciente. Además, otro fundamento importante a considerar en el plan de tratamiento en este caso fue que el RI permite mantener volumen del hueso alveolar, hasta que el paciente termine su crecimiento, y favorecer una adecuada calidad de vida con la permanencia de sus dientes en boca en un periodo importante del desarrollo como es la pubertad y la adolescencia.

\section{CONCLUSIONES}

La recuperación de la estética y función en adolescentes con FCR en la zona anterior representa un complejo desafío para el cirujano dentista, quien debe estar preparado y en constante actualización para lograr entregar el mejor tratamiento posible. 
El reimplante intencional junto con la reposición extraoral del fragmentos con técnicas adhesivas para el manejo y solución de fracturas corono radiculares en incisivos permanentes, puede ser una adecuada alternativa terapéutica conservadora y mínimamente invasiva de buena relación costo efectividad en el paciente infantil o adolescente, esto gracias a la gran capacidad de cicatrización del LPD, y al desarrollo de técnicas de adhesión con los biomateriales existentes actualmente.
Un adecuado y razonable manejo de la urgencia es fundamental a la hora de planificar un tratamiento definitivo. De acuerdo a lo observado en 16 meses de seguimiento, no se observan signos de reabsorción por reemplazo ni reabsorción inflamatoria en el diente 11 , se mantiene una condición fisiológica, y una estética aceptable. Pacientes adolescentes afectados con este tipo de traumatismo dentario debe mantener controles clínicos y radiográficos a largo plazo, especialmente mientras no terminen su etapa de crecimiento.

DÍAZ, M. J.; BARRERA, O. V.; JANS, M. A. \& ZAROR, S. C. Clinical approach of permanent maxillary incisor with complicated crown-root fracture through intentional replantation. Int. J. Odontostomat., 8(2):299-307, 2014.

ABSTRACT: Crown root fracture management is a clinical and interdisciplinary challenge, either in its initial emergency care as in the definitive treatment planning and execution. Three main treatment alternatives are described: surgical and orthodontic extrusion of crown-root remnant, gingivectomy and intentional reimplantation, depending on factors such as: age of patient, clinical conditions of periodontal ligament and root length. This paper reports a clinical case of an adhesive fragment reattachment in a complicated crown root fracture in upper maxillary central incisor through intentional replantation. A 10 year-old male patient with complex dentoalveolar trauma, which highlights a complicated crown root fracture on tooth 11 with subgingival extension greater than $5 \mathrm{~mm}$. Given the complexity of the clinical situation, we opted for intentional replantation, to allow reattachment of the crown fragment by adhesive technique. After the first sixteen months of follow-up, an adequate periodontal healing, acceptable aesthetic and functional outcome were observed. The intentional replantation can be considered adequate treatment in cases of complex crown root fractures when the fracture feature is located in areas that prevent the use of other techniques for rehabilitation. In the same way it allows the maintenance of the height and volume of the alveolar bone of growing patients.

KEY WORDS: tooth replant, crown-root fractures in children, crown fragment re-attachment.

\section{REFERENCIAS BIBLIOGRÁFICAS}

Andreasen, J. O. \& Hjorting-Hansen, E. Replantation of teeth. I. Radiographic and clinical study of 110 human teeth replanted after accidental loss. Acta Odontol. Scand., 24(3):263-86, 1966.

Andreasen, J. O.; Andreasen, F. M. \& Andersson, L. Texto y Atlas a Color de Lesiones Traumáticas a las Estructuras Dentales. $4^{\text {a }}$ ed. Caracas, Amolca, 2010.

Baratieri, L. N.; Monteiro Júnior, S.; Cardoso, A. C. \& de Melo Filho, J. C. Coronal fracture with invasion of the biologic width: a case report. Quintessence Int., 24(2):85-91, 1993.

Cortes, M. I.; Marcenes, W. \& Sheiham, A. Impact of traumatic injuries to the permanent teeth on the oral health-related quality of life in 12-14-year-old children. Community Dent. Oral Epidemiol., 30(3):193-8, 2002.

Czochrowska, E. M.; Stenvik, A.; Bjercke, B. \& Zachrisson, B. U. Outcome of tooth transplantation: survival and success rates 17-41 years posttreatment. Am. J. Orthod. Dentofac. Orthop., 121(2):110-9, 2002.
Demarco, F. F.; Fay, R. M.; Pinzon, L. M. \& Powers, J. M. Fracture resistance of re-attached coronal fragments-influence of different adhesive materials and bevel preparation. Dent Traumatol., 20(3):157-63, 2004.

Diangelis, A. J.; Andreasen, J. O.; Ebeleseder, K. A.; Kenny, D. J.; Trope, M.; Sigurdsson, A.; Andersson, L.; Bourguignon, C.; Flores, M. T.; Hicks, M. L.; Lenzi, A. R.; Malmgren, B.; Moule, A. J.; Pohl, Y.; Tsukiboshi, M. \& International Association of Dental Traumatology. International Association of Dental Traumatology guidelines for the management of traumatic dental injuries: 1 . Fractures and luxations of permanent teeth. Dent. Traumatol., 28(1):2-12, 2012.

Dogan, M. C.; Akgun, E. O. \& Yoldas, H. O. Adhesive tooth fragment reattachment with intentional replantation: 36month follow-up. Dent. Traumatol., 29(3):238-42, 2013.

Fariniuk, L. F.; Ferreira, E. L.; Soresini, G. C.; Cavali, A. E \& Baratto Filho, F. Intentional replantation with 180 degrees rotation of a crown-root fracture: a case report. Dent. Traumatol., 19(6):321-5, 2003. 
Glendor, U. Epidemiology of traumatic dental injuries--a 12 year review of the literature. Dent. Traumatol., 24(6):60311, 2008.

Glendor, U. Aetiology and risk factors related to traumatic dental injuries--a review of the literature. Dent. Traumatol., 25(1):19-31, 2009.

Kim, D. S.; Shin, D. R.; Choi, G. W.; Park, S. H.; Lee, J. W. \& Kim, S. Y. Management of complicated crown-root fractures using intentional replantation: two case reports. Dent. Traumatol., 29(4):334-7, 2013.

Marcenes, W.; Alessi, O. N. \& Traebert, J. Causes and prevalence of traumatic injuries to the permanent incisors of school children aged 12 years in Jaragua do Sul, Brazil. Int. Dent. J., 50(2):87-92, 2000.

de Castro, M. A.; Poi, W. R.; de Castro, J. C.; Panzarini, S. G.; Sonoda, C. K.; Trevisan, C. L. \& Luvizuto, E. R. Crown and crown-root fractures: an evaluation of the treatment plans for management proposed by 154 specialists in restorative dentistry. Dent. Traumatol., 26(3):236-42, 2010.

Oesterle, L. J. \& Wood, L. W. Raising the root. A look at orthodontic extrusion. J. Am. Dent. Assoc., 122(7):1938, 1991.

Park, J. H.; Tai, K. \& Hayashi, D. Tooth autotransplantation as a treatment option: a review. J. Clin. Pediatr. Dent., 35(2):129-35, 2010.

Peer, M. Intentional replantation - a 'last resort' treatment or a conventional treatment procedure? nine case reports. Dent. Traumatol., 20(1):48-55, 2004.

Ramos-Jorge, M. L.; Bosco, V. L.; Peres, M. A. \& Nunes, A. C. The impact of treatment of dental trauma on the quality of life of adolescents - a case-control study in southern Brazil. Dent. Traumatol., 23(2):114-9, 2007.

Rapelli, G.; Massaccesi, C. \& Putignano, A. Clinical procedures for the immediate reattachment of a tooth fragment. Dent. Traumatol., 18(5):281-4, 2002.

Saito, C. T.; Guskuma, M. H.; Gulinelli, J. L.; Sonoda, C. K.; Garcia-Júnior, I. R.; Filho, O. M. \& Panzarini, S. R. Management of a complicated crown-root fracture using adhesive fragment reattachment and orthodontic extrusion. Dent Traumatol., 25(5):541-4, 2009.

Sockalingam, S. N. M. P. \& Mahyuddin, A. Complicated crown root fracture treatment option: a case report. Arch. Orofac. Sci., 4(1):25-8, 2009.

Traebert, J.; Telino de Lacerda, T.; Foster Page, L. A.; Thomson, W. M. \& Bortoluzzi, M. C. Impact of traumatic dental injuries on the quality of life of schoolchildren. Dent Traumatol., 28(6):423-8, 2012.

Tsukiboshi, M. Autotransplantation of teeth: requirements for predictable success. Dent. Traumatol., 18(4):157-80, 2002.

Zachrisson, B. U.; Stenvik, A. \& Haanæs, H. R. Management of missing maxillary anterior teeth with emphasis on autotransplantation. Am. J. Orthod. Dentofac. Orthop., 126(3):284-8, 2004

Dirección para Correspondencia:

Jaime Díaz Meléndez

Director, Especialidad de Odontopediatría

Facultad de Odontología

Universidad de la Frontera

Claro Solar 115, Temuco

CHILE

Email: jaime.diaz@ufrontera.cl

Recibido: 07-10-2013

Aceptado: 27-06-2014 